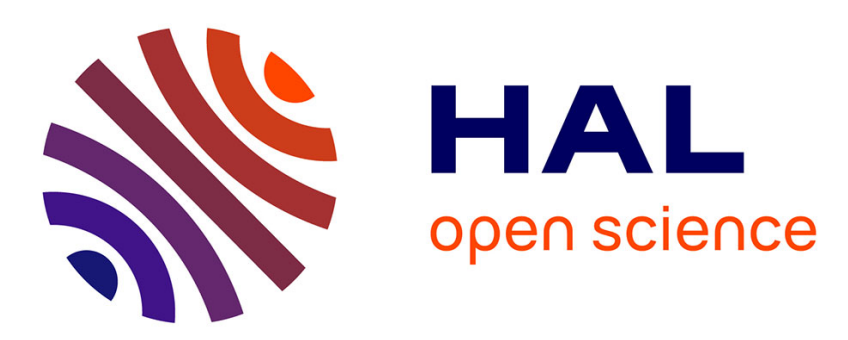

\title{
Caractérisation de quelques structures et architectures forestières des arbres et arbustes à feuilles persistantes de l'étage méditerranéen
}

Marcel Barbero

\section{- To cite this version:}

Marcel Barbero. Caractérisation de quelques structures et architectures forestières des arbres et arbustes à feuilles persistantes de l'étage méditerranéen. Revue forestière française, 1988, 60 (5), pp.371-380. 10.4267/2042/25904 . hal-03424814

\section{HAL Id: hal-03424814 \\ https://hal.science/hal-03424814}

Submitted on 10 Nov 2021

HAL is a multi-disciplinary open access archive for the deposit and dissemination of scientific research documents, whether they are published or not. The documents may come from teaching and research institutions in France or abroad, or from public or private research centers.
L'archive ouverte pluridisciplinaire HAL, est destinée au dépôt et à la diffusion de documents scientifiques de niveau recherche, publiés ou non, émanant des établissements d'enseignement et de recherche français ou étrangers, des laboratoires publics ou privés. 


\title{
CARACTÉRISATION DE QUELQUES STRUCTURES \\ ET ARCHITECTURES FORESTIĖRES DES ARBRES ET ARBUSTES À FEUILLES PERSISTANTES DE L'ÉTAGE MÉDITERRANÉEN
}

\author{
M. BARBERO
}

\section{AVANT-PROPOS}

Les écosystèmes forestiers méditerranéens sont constitués pour l'essentiel par des végétaux dont les organes foliaires persistants sont adaptés de différentes manières (cuticule épaisse, feuilles vernissées, tomenteuses) à la sécheresse estivale et que l'on qualifie de sclérophylles. Ils sont, par ailleurs, caractérisés dans la distribution de leurs constituants principaux par une forte hétérogénéité dont l'étude a nécessité la mise au point de stratégies d'échantillonnages spécifiques. Tel a été le cas en particulier pour les chênaies sclérophylles (Miglioretti, 1983, 1987).

En effet, quelles que soient les questions de recherche posées, l'approche de ces systemes hétérogènes doit toujours être réalisée sur une base de stratification des échantillons étudiés, tenant compte des échelles et des niveaux d'investigations.

Cette démarche est d'autant plus nécessaire que la méthode phytosociologique la plus anciennement employée pour l'étude des structures forestières a conduit à décrire de grandes associations-cadre [Quercetum ilicis galloprovinciale Br.-BI. (1915) 1936, Quercetum mediterraneo-montanum Br.-BI. 1936, Querco-Buxetum Br.-BI. (1931) 1936], représentant des aires de relevés homogènes mais variant, pour chaque formation, dans l'espace et dans le temps (Vignes, 1980). Ce concept d'homogénéité a cependant amené à simplifier quelque peu l'état des structures "in natura " et à masquer l'hétérogénéité des grandes formations végétales forestières. L'approche choisie ne répondait pas ainsi aux questions que se posaient d'autres chercheurs, mais aussi les gestionnaires qui, à partir de la simple observation de terrain, constataient que les ensembles forestiers méditerranéens étaient en fait très complexes et très hétérogènes.

Travail dédié à notre collègue et ami Debazac. 


\section{BARBERO}

Un tel constat était encore amplifié par les questions relatives à la signification phytoécologique des nombreuses structures préforestières à conifères qui caractérisent les paysages méditerranéens fortement perturbés.

Il en était ainsi des pinèdes de Pin d'Alep, de Pin mésogéen, de Pin pignon qui occupent une place majeure dans le tapis végétal arborescent et dont les essences principales sont présentes en colonisations de forts recouvrements à tous les stades de la succession (modèle expansionniste). À leur niveau, il est très difficile de recenser toutes les unités structurales tant elles sont nombreuses et variées en fonction des régions, stations et placettes.

De tout cela, il résulte que les recherches enı écologie de l'hétérogénéité doivent trouver des bases conceptuelles et méthodologiques spécifiques, surtout en région méditerranéenne oủ dominent les systèmes complexes auxquels appartiennent les ensembles forestiers d'où l'effort méthodologique entrepris sur les taillis (Miglioretti, 1983).

En fait, l'écologie du paysage forestier vise à donner un contenu rationalisé aux concepts de stabilité, homogénéité, hétérogénéité, diversité, qui permettent d'asseoir l'étude thèorique des mécanismes de structuration et de fonctionnement des écosystèmes forestiers stabilisés (Odum, 1969), métastables (Godron, 1984) et du non-équilibre parce que entretenus dans leur état temporel par les perturbations (Grime, 1977 ; Pickett, 1982) qui modifient les tendances prévisibles de la dynamique spatiale.

Les écosystèmes forestiers stabilisès (climaciques) qui reprèsentent l'état d'équilibre de la végétation, du sol et du climat dans une région donnée ne sont pratiquement jamais réalisés en région méditerranéenne. S'observent en effet des écosystèmes métastables qui constituent, dans des conditions stationnelles déterminées, l'évolution optimale d'un ècosystème forestier, compte tenu des contraintes locales du milieu et des caractéristiques biologiques de l'essence forestière dominante. Les écosystèmes forestiers du non-équilibre fréquents en région méditerranéenne (pinèdes de Pin d'Alep par exemple) forment quant à eux des structures préforestières peu évoluées où les espèces typiquement liées aux écosystèmes forestiers sont rares en raison de l'occurrence régulière des perturbations (incendies par exemple). Dominent dans leur sousbois des espèces arbustives des stades régressifs des successions (ligneux des maquis et garrigues).

\section{BASES RATIONNELLES DE L'ĖTUDE DES STRUCTURES}

L'analyse des structures forestières fait appel aux critères de densités spécifiques et interspécifiques avec, dans ce cas précis, une approche de la diversité dans la globalité de l'écosystème forestier ou au sein d'une seule strate.

En zone forestière méditerranéenne plus qu'ailleurs encore, cette analyse doit être subordonnée d'emblée à la prise en compte de la variation rapide des paramètres très hétérogènes du milieu et donc de la composition des peuplements.

II ne faut pas oublier d'intégrer aussi les résultats de l'enquête cadastrale préalable sur les parcelles étudiées qui sont absolument nécessaires pour aborder les problèmes historiques passés ou récents de la région, de la station et de la placette.

\section{Les paramètres abiotiques}

\section{- L'hétérogénéité bioclimatique}

La très forte variation des conditions climatiques est une des caractéristiques de la règion méditerranèenne. Emberger (1942), dans son diagramme pluviothermique, avait mis en évidence 
cette particularité et toutes les nombreuses études, qui en ont découlé et qui continuent encore, donnent à la caractérisation physique du climat et à ses variations annuelles et interannuelles une fonction biologique fondamentale.

Cette hétérogénéité détermine sur les écosystèmes, à l'échelle régionale, les successions altitudinales et latitudinales par grands ensembles bioclimatiques. Ces derniers caractérisés à partir d'une analyse des structures forestières potentielles représentent dans une région donnée au terme de la sylvigénèse les ensembles de composition floristique les plus stables en fonction notamment des caractéristiques édaphiques stationnelles. Ils sont fortement réduits spatialement compte tenu des perturbations que ces écosystèmes subissent (Demangeot, 1984). IIs n'en traduisent pas moins, par leur adaptation au climat, l'hétérogénéité bioclimatique méditerranéenne: grandes variations à l'échelle géographique de la moyenne des minimums du mois le plus froid $(\mathrm{m})$, forte variabilité des précipitations annuelles et interannuelles, durée de la sécheresse.

Pour les structures des yeuseraies par exemple, l'organisation est très complexe (figure 1). Elle s'éloigne très largement des schémas classiques avancés par Braun-Blanquet (1952). Plusieurs ensembles ont été décrits récemment et notamment l'Epipactido-Quercetum ilicis (Barbero et Loisel, 1983) et le Piptathero-Quercetum ilicis (Quezel et Barbero, 1986).

En outre, l'hẻtérogénèité climatique doit être abordèe à la fois aux niveaux stationnel et temporel avec les analyses fréquentielles et interannuelles qui permettent de bien comprendre le déterminisme de la répartition spatiale de certains peuplements forestiers. Cette hétérogéneité bioclimatique doit être aussi plus directement exprimée par la prise en compte des réponses aux contraintes comme en particulier le stress climatique lié d'une part à la sécheresse estivale, à

Figure 1

\section{STRUCTURES DES CHÊNAIES SCLÉROPHYLLES}

\section{ÉTAGE MÉSOMÉOITERRANÉEN}

\section{Niveau inférieur}

Bioclimat subhumide tempéré

- yeuseraie à Laurus nobilis, Pistacia lentiscus (Lauro-Quercetum ilicis)

- yeuseraie à Celtis australis (Celtido-Quercetum ilicis)

\section{Niveau supérieur}

Bioclimat subhumide frais

- yeuseraie à Orchidèes d'affinités ibéro-provençales (Epipactido-Quercetum ilicis, Piptathero-Quercetum ilicis)

Bioclimat humide frais

- yeuseraie à Charme-houblon d'affinités liguro-tyrrhéniennes (Orno-Quercetum ilicis ostryetosum)

\section{ÉTAGE MÉDITERRANÉEN SUPÉRIEUR}

Sur silice; bioclimat humide froid

- yeuseraies acidophiles à llex aquifolium (sommet des Maures, Cévennes)

Sur calcaire ; bioclimat subhumide froid

- yeuseraie calcaricole à If

- yeuseraie calcaricole à Buis

Chênaies pubescentes méditerranéennes

\section{SUPRAMÉDITERRANÉEN}

Chênaies pubescentes

à Buis et Acer opalus 


\section{BARBERO}

son intensité, à sa durée et d'autre part aussi à l'action du vent et plus particulièrement au mistral qui impose aux essences majeures de résister à l'évapotranspiration.

Or, la structure forestière méditerranéenne la mieux adaptée à ces situations est le modèle sclérophylle par son aptitude à s'adapter notamment dans les secteurs de très fortes contraintes et de très faible concurrence interspécifique: falaises, crêtes à toutes les expositions et versants à climat contrasté.

Il faut remarquer d'ailleurs que ce modèle occupe aussi les zones bien protégées où cependant les différentes espèces de sclérophylles et en particulier le Chêne vert sont fortement concurrencées par d'autres essences comme le Chêne pubescent.

Ce modèle est donc constitué d'éléments dont les stratégies adaptatives au climat sont très fortes: résistance au stress hydrique, aptitude à s'adapter à des situations de lumière très contrastées. Les mécanismes écophysiologiques de l'adaptation se répercutent au niveau du système foliaire (index de surface, épaisseur, teneur en eau variable suivant les expositions et les strates de l'arbre) (Madjidieh, 1987 ; Baldy, Barbero, Madjidieh, Quezel, 1987).

\section{- Hétérogénéité géopédologique}

Compte tenu des fortes perturbations anthropozoogènes que les écosystèmes méditerranéens ont subies, il est difficile de décrire à leur niveau, mis à part dans quelques structures très évoluées, des sols d'équilibre. Les recherches engagées par Callot et par nous-mêmes et poursuivies depuis par Aubert (1984-1985) ont montré la très forte variation spatiale des complexes édaphiques méditerranéens.

En effet, en région méditerranéenne, il est préférable d'envisager l'approche de l'hétérogénéité édaphique non sous l'angle du sol proprement dit, mais sous celui de la relation sol-substrat. La structure géopédologique stationnelle doit donc être conceptualisée comme une entité, à laquelle il convient d'agréger des paramètres comme la pente, le pendage ou le contre-pendage des couches, le mode de fragmentation de la roche, la réserve de terre fine. Toutes ces caractéristiques variables dans l'espace permettent d'appréhender la dynamique de l'eau dans le sol, son stockage et donc d'expliquer certains éléments des caractéristiques de la structure forestière que le modèle géopédologique porte.

Il est donc sûr que la nature de l'humus, son statut en bases qui jouent un rôle capital dans l'économie de l'eau de surface dans les grands écosystèmes forestiers médioeuropéens n'a pas ici, en région méditerranéenne, la même importance et, de ce fait, la caractérisation de groupes écologiques à partir de l'état d'équilibre de l'humus dans les sols (Duvigneaud, 1980 ; Duchaufour, 1960 ; Ellenberg, 1979) n'est possible que pour quelques structures d'écosystèmes forestiers très évolués ou métastables.

Là encore le "type sclérophylle" apparaît par rapport au modèle à feuillage caduc comme plus agressif et plus adapté aux milieux à fortes contraintes géopédologiques (modèle de résistance).

Il en est ainsi pour le Chêne vert qui, à l'instar d'autres sclérophylles de mêmes types étudiés par Miller (1982), colonise les sols à complexe absorbant déficient et donc à fort déficit ionique, ce que ne peut pas réaliser par exemple le Chêne pubescent qui est beaucoup plus exigeant lorsqu'il y a carence en cations et anions principaux du sol. Le type sclérophylle reste néanmoins, malgré la concurrence des Chênes à feuillage caduc, adapté aux conditions de luxe trophique, ce qui permet d'expliquer la large répartition spatiale et la très forte hétérogénéité des structures géopédologiques que ce Chêne colonise (Miglioretti et Barbero, 1984).

Cette adaptation à la contrainte trophique très forte chez les sclérophylles comme le Chêne vert l'est encore davantage chez les conifères méditerranéens comme le Pin d'Alep qui représente le modèle type des essences colonisatrices expansionnistes fortement adaptées aux milieux pau- 
vres et capables d'ajuster rapidement la démographie de leurs peuplements à la contrainte. A la différence du Chêne vert, le Pin d'Alep apparaît dans tous les stades de la successioir, depuis les groupements pionniers où il était remarquablement adapté à la compétition par exploitation. jusqu'aux stades terminaux où il tolère peu la compétition par interférence; il s'adapte mal à celle-ci en ne régénérant pas et en étant fortement concurrencé par les Chênes sclérophylles ou caducifoliés. Ainsi se sont constituées toutes les structures mixtes observables en Provence sur les parcelles les plus anciennement abandonnées et épargnées jusqu'ici des perturbations par incendies (pinèdes à sous-étage de Chêne vert et de Chêne pubescent).

\section{Les paramètres biologiques}

\section{- Paramètres endogènes}

IIs conditionnent par les caractéristiques propres à chaque essence forestiere le déterminisme d'organisation des modèles structuraux et architecturaux des peuplements et leur adaptation aux contraintes précédemment définies.

C'est dire combien les recherches sur les stratégies adaptatives dans les domaines de la régénération, de la démographie des essences forestières, de la biologie de la reproduction et de l'écophysiologie jouent un rôle essentiel. Les sclérophylles dominants de la végétation méditerranéenne présentent, en règle générale au sein des peuplements, des décalages phénologiques temporels importants. Les sclérophylles dominants de la végétation méditerranéenne sont souvent allogames: par des décalages phénologiques temporels importants dans la pollinisation entre individus souvent proches spatialement, ils assurent entre eux des croisements à distance et donc des échanges géniques continus et diversifiés s'exprimant plus particulièrement par une reconstitution rapide de la diversité génétique spatiale leur assurant d'emblée une meilleure réussite par rapport à d'autres essences (Yacine, 1987).

Ces modèles sont aussi bien adaptés aux stress avec, en particulier pour eux. le choix de stratégies de survie dans les situations de fortes perturbations climatiques. Par exemple, lors des périodes de sécheresse de longue durée, comme les connaît certaines années la végétation méditerranéenne, l'arbre, suivant les situations d'hétérogénéité climatique, se défend et survit par des stratégies appropriées : d'abord élimination des teuilles de deux ans, chute des fruits et maintien d'un nombre réduit de feuilles de l'année pour assurer une activité photosynthètique minimale (Madjidieh, 1987).

Ainsi apparaît clairement la nécessité de la prise en compte de l'écogénétique pour la compréhension de la biologie des populations et des structures à Chênes sclérophylles méditerranéens.

\section{- Paramètres exogènes}

L'évolution des structures au cours des diverses étapes de la sylvigènèse est conditionnée non seulement par les caractéristiques propres à l'essence et en particulier les stratégies écophysiologiques d'adaptation à la lumière, mais encore par les actions biologiques et en particulier les vecteurs de la dissémination des espèces dans les structures sous l'action du vent (anémochorie) et des animaux (zoochorie).

L'évolution actuelle des peuplements à sclérophylles dans les zones privées de perturbations depuis quelques décennies est marquée par la montée en puissance des végétaux dont les graines sont disséminées par les oiseaux (ornithochorie) et pour lesquels les individus sont adaptés aux conditions de faible lumière (sciaphilie) et dont les baies et les fruits sont largement disséminés : Laurier noble, Lierre, Viorne-tin, Houx-fragon, Garance sauvage, Filaria.

Ici encore la très forte hétérogénéité spatiale des individus de structures doit être reliée à l'hétérogénéité de la répartition des populations animales présentes à leur voisinage (Escarre, Houssard, Debussche, 1983). 


\section{Les données socio-historiques}

Les bases historiques sont fondamentales pour comprendre les caractéristiques actuelles des écosystèmes forestiers méditerranéens. Différentes échelles de temps servent de support à l'approche historique, que ce soit au niveau de l'analyse pollinique (palynologie) (Pons et Vernet, 1971), que l'étude et la datation des microrestes de charbons contenus dans les horizons des so!s (pédoanthracologie) (Thinon, 1979). Mais sont très importantes aussi toutes les analyses et enquêtes historiques plus récentes et particulièrement axées sur les documents cadastraux.

L'approche cadastrale permet, en effet, par le relais avec la parcelle, d'appréhender la vitesse d'installation et de cicatrisation d'une structure après abandon d'usage ou après une perturbation comme la coupe.

Plusieurs approches de ce type réalisées au Laboratoire d'Écologie méditerranéenne (Greco CNRS, 1986) - communes de Rocbaron (83), Vidauban (83), Levens (06) - montrent que, si la plupart des structures à Pin d'Alep sont héritées de systèmes cultivés encore récemment, beaucoup de yeuseraies, plus anciennes au contraire, se sont installées sur des parcelles qui, durant la première moitié du XIX ${ }^{\complement}$ siècle, étaient occupées par les olivettes; il n'est pas rare de retrouver dans tous ces écosystèmes à sclérophylles des rejets d'Oléastre.

Le mode de gestion de l'écosystème est aussi une dimension capitale de sa structuration. L'analyse comparative dans une même région entre forêts domaniales, communales, soumises ou non soumises au régime forestier et privées, permet de mieux mesurer le poids de la gestion raisonnée dans l'expression des potentialités actuelles des structures forestières méditerranéennes et de bien aborder aussi le déterminisme de leur hétérogénéité et de leur diversité. Cette analyse a été menée à bien en comparant, par exemple, dans des conditions écologiques identiques, des parcelles forestières soumises de la forêt domaniale à des parcelles de la forêt communale non soumise de Rians (83). Les premières montrent une remarquable remontée biologique avec le développement dans la strate herbacée des écosystèmes d'un lot important d'espèces du mull-forestier (Miglioretti et Barbero, 1984).

Enfin, l'analyse comparative de documents phytoécologiques réalisés à des pas de temps variables est capitale pour comprendre la sylvigénèse de nombreuses structures de végétation forestière méditerranéenne à sclérophylles.

$\mathrm{Si}$, en effet, par l'analyse synchronique, il n'est pas difficile, pour les structures de colonisation préforestières comme les pinèdes de Pin d'Alep, de prendre en compte le critère âge pour les positionner aux différents stades de la succession, le problème est autrement plus complexe dans les structures potentielles dont les essences majeures rejettent de souche.

L'existence au Laboratoire de Botanique et d'Écologie méditerranéenne d'une Phytothèque, constituée dès les années 1964, permet aujourd'hui en étudiant les mêmes structures là où elles n'ont pas été perturbées, d'avoir une bonne idée de leur maturation temporelle.

Il est démontré par exemple que les chênaies vertes thermophiles à Laurier noble se transforment, à des pas de temps de deux décennies, par le développement d'une strate herbacée à Lierre et à Fougères - Asplenium trichornanes, Asplenium onopteris, Polystrichum aculeatum et aussi par l'installation d'autres espèces comme Carex grioletti, Ruscus hypophyllum et même Pteris cretica dans les Alpes maritimes.

L'évolution du Viburnum-Quercetum ilicis (figure 2) se fait vers une structure où le Lierre est important et où apparaissent de nombreuses Orchidées hémisaprophytes liées à une matière organique de litière à décomposition lente: Epipactido-Quercetum ilicis (Barbero et Loisel, 1983).

Le remplacement de l'Orno-Quercetum ilicis terebinthosum dans les Alpes maritimes et ligures par une structure fortement enrichie en espèces caducifoliées est spectaculaire dans cette 


\begin{tabular}{|c|c|c|c|}
\hline \multicolumn{2}{|c|}{ PROVENCE } & \multicolumn{2}{|c|}{ ALPES MARITIMES } \\
\hline$\left\{\begin{array}{l}\text { Groupements arbustifs } \\
\text { et préforestiers }\end{array}\right.$ & $\left\{\begin{array}{c}\text { Ourlets, haies } \\
\text { ensembles } \\
\text { préforestiers }\end{array}\right.$ & $\left\{\begin{array}{l}\text { Groupements arbustifs } \\
\text { et prëforestiers }\end{array}\right.$ & {$\left[\begin{array}{c}\text { Ourlets, haies } \\
\text { ensembles } \\
\text { preforestiers }\end{array}\right.$} \\
\hline $\left.\begin{array}{l}\text { Quercetum cocciferae } \\
\text { Rosmarino-Lithospermetum } \\
\text { Staehelino-Dorycnietum } \\
\text { avec ou sans Pin d'Alep }\end{array}\right\}$ & $\begin{array}{c}\text { Clematido- } \\
\text { Pistacietum } \\
\text { terebinthi } \\
\downarrow\end{array}$ & $\begin{array}{l}\text { Staehelino-Dorycnietum } \\
\text { Genistetosum cinereae } \\
\text { Rosmarinetosum } \\
\text { avec ou sans Pin d'Alep }\end{array}$ & $\begin{array}{l}\text { Coriario-Cotineto } \\
\text { Quercetum ilicis } \\
\qquad\end{array}$ \\
\hline Forêt prèpotentielle & $\begin{array}{c}\text { Viburno-Quercetum } \\
\text { ilicis } \\
\downarrow\end{array}$ & Forêt prépotentielle & $\begin{array}{l}\text { Orno-Quercetum } \\
\text { ilicis terebinthosum }\end{array}$ \\
\hline Forêt potentielle & $\begin{array}{l}\text { Epipactido- } \\
\text { Quercetum ilicis }\end{array}$ & Forêt potentielle & $\begin{array}{l}\text { Orno-Quercetum } \\
\text { ilicis ostryetosum }\end{array}$ \\
\hline
\end{tabular}

région où les précipitations sont plus importantes qu'en Provence; Charme-houblon, Chêne pubescent, Alisier blanc, Frêne à fleurs sont les essences les plus fréquemment associées au Chêne vert dans l'Orno-Quercetum ilicis ostryetosum.

Ces résultats dèmontrent à l'évidence la difficulté de retenir le concept de climax dans les structures forestières méditerranéennes actuelles. Les études d'écologie diachronique attestent que, dans les peuplements à sclérophylles, on est loin d'avoir atteint le potentiel réel; la plupart des peuplements sont en situation de métastabilité. Les tendances de l'évolution actuelle portent presque partout "in natura" vers la constitution à l'étage méditerranéen de forêts mélangées qui permettent de donner une idée de ce que devaient être les forêts initiales beaucoup plus diversifiées encore dans leur strate arborescente que la plupart des peuplements à sclérophylles d'aujourd'hui.

Les structures et architectures forestières de forêts de Chêne vert depuis longtemps protégées comme celle de Valbonne (30) permettent d'en rendre compte.

\section{MODĖLES DYNAMIQUES ET ARCHITECTURAUX}

Toutes les recherches en écologie forestière convergent vers la prise en consideration:

- de la dynamique des structures forestières complexes et hétèrogènes se succédant dans l'espace et dans le temps;

- des particularités stationnelles de ces systèmes complexes et leur caractérisation nécessaire par des paramètres simples évaluables sur le terrain et d'ordre qualitatif, semi-quantitatif et quantitatif. Parmi ces paramètres simples, la composition spécifique, les recouvrements appréhendès par la méthode des transects permettent d'approfondir par l'analyse comparée les concepts de diversité spécifique et maximale et la définition de gradients au cours des diffèrentes ètapes de la sylvigénèse depuis les ensembles préforestiers jusqu'aux unités forestières. 
Cette étude a conduit à élaborer de nouveaux concepts pour caractériser la Chênaie verte dont l'hétérogénéité est telle que plusieurs modèles dynamiques ont dû être définis dans l'espace et dans le temps (cf. figure 2, page 377).

L'ëtude de ces modèles complexes couplée avec celle des paramètres dendromètriques, euxmêmes reliés aux caractéristiques abiotiques, débouche sur la notion de typologie synthétique principalement axée sur la caractérisation de groupes écodendrométriques, centrés sur les interrelations floristique, dendrométrique et écologique.

Parallèlement à l'analyse des structures, la caractérisation de modèles architecturaux est possible:

- en forêt pure pour l'essence dominante,

- en forêt mélangée pour les types forestiers codominants,

- pour l'écosystème lui-même aux différentes ètapes de son évolution.

Dans les systèmes de taillis comme l'ont montré Miglioretti et Barbero (1984), les fonctions de réponse des cépées sclérophylles dans l'espace sont directement reliées aux structures géopèdologiques des stations. Plusieurs modèles (figure 3) peuvent être dècrits sur la base de ces critères pour des taillis exploités à une même époque:

- un modèle architectural progressif dans les stations où les densités de cépées sont faibles. Les brins sont nombreux et coexistent depuis les positions subhorizontales jusqu'aux positions verticales;

Figure 3

MODĖLES ARCHITECTURAUX DES TAILLIS MÉDITERRANÉENS

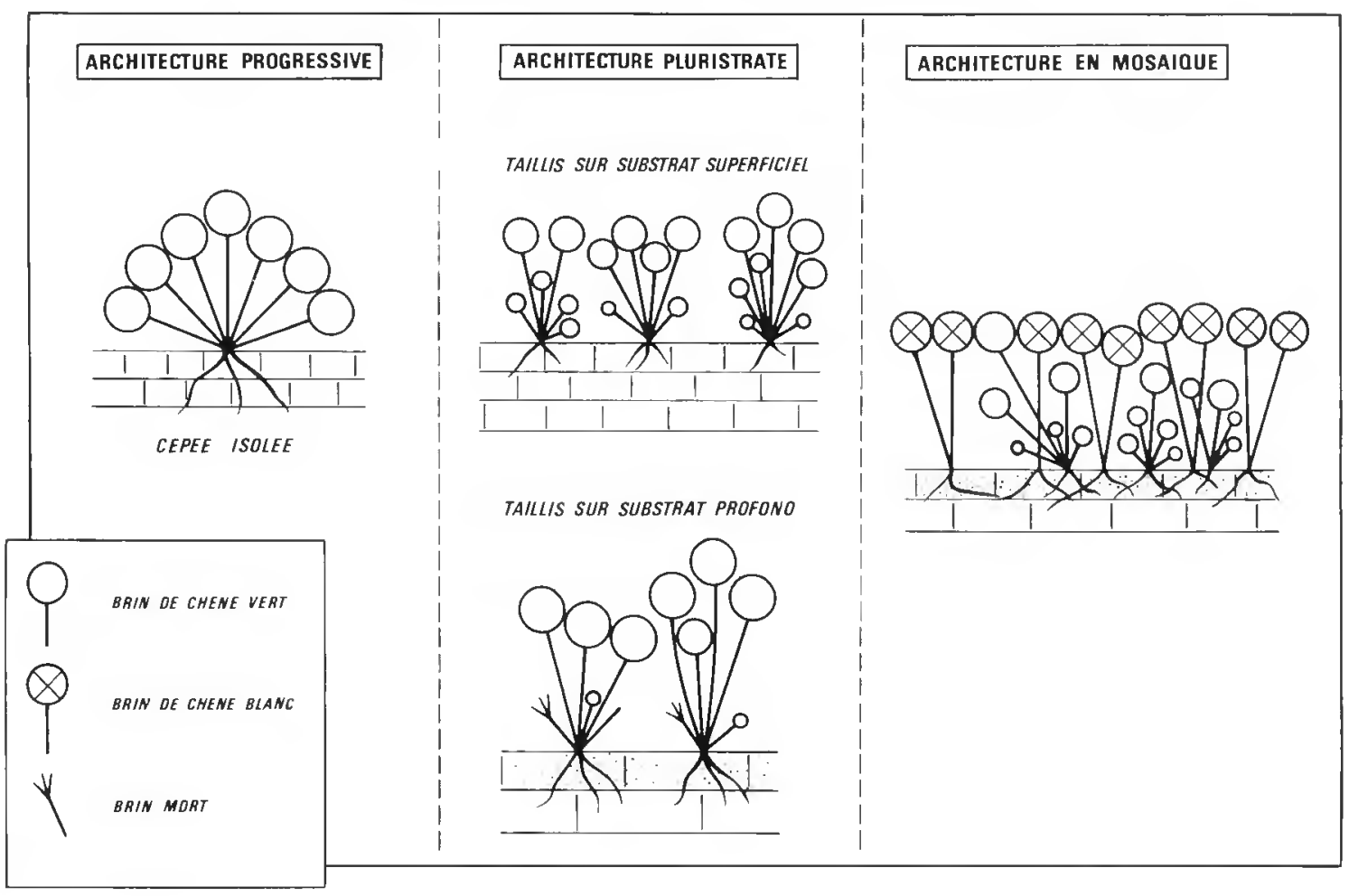


- un modèle architectural pluristrates avec des brins nettement dominants et des brins dominés qui seront rapidement éliminés dans les stations de bonnes potentialités:

- des modèles architecturaux de mosaïques où, en concurrence interspécifique, les feuillus sclérophylles et à feuillage caduc se partagent en densités de cépées à peu près équivalentes le bio-volume. Les modèles d'évolution temporelle placent le Chêne vert en situation d'arbre dominé dans la sous-strate arborescente et quelquefois la strate arbustive. II réagit en émettant des brins portant des feuilles à index de surface foliaire élevé pour compenser l'extinction du rayonnement lumineux (Baldy, Barbero, Madjidieh, 1987).

\section{CONCLUSIONS}

Toutes ces recherches basées sur l'analyse spatio-temporelle de l'hétérogénéité visent donc à aborder d'une façon à la fois synthétique et fonctionnelle la caractérisation des structures et architectures forestières de la végétation de l'étage méditerranéen.

Elles font ressortir clairement la nécessité d'une approche progressive, conceptuelle et théorique, par échelles et niveaux, qui permet de mieux quantifier les principaux paramètres et variables dont la prise en compte est nécessaire pour parvenir à une typologie synthétique des stations.

C'est le seul moyen de définir une politique raisonnée de gestion assise sur un coût écologique minimal et qui assure en même temps une prévention efficace contre les perturbations majeures que subissent ces écosystèmes à la faveur d'incendies.

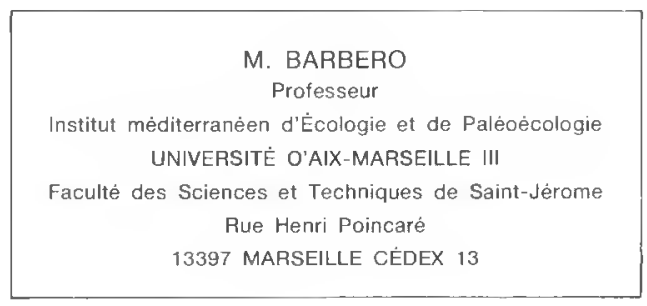

\section{BIBLIOGRAPHIE}

AUBERT (G.). - Guide écologique de la forêt domaniale de la Gardiole (Massif des Calanques (13), France). La végétation et ses relations avec les facteurs pédologiques. - Marseille : Centre régional de Documentation pédagogique ; Centre national de Documentation pédagogique, 1984-1985. - Tome 1, 69 p. ; tome 2, 38 p.

BALDY (Ch.), BARBERO (M.), MADJIDIEH (H.), OUEZEL (P.). - Caracteristiques biophysiques foliaires du modèle sclérophylle en situation de stress hydrique. Le cas du Chêne vert. - Collection Medekos, juillet 1987 (sous presse).

BARBERO (M.), LOISEL (R.). - Les chênaies du Sud-Est de la France méditerranéenne. Valeurs phytosociologiques, dynamiques et potentielles. - Phytocoenologia, vol. 2, $n^{\circ} 2,1983$, pp. 225-244.

BRAUN-BLANQUET (J.). - La chênaie d'Yeuse méditerranéenne. - Mémoire de la Société d'Études des Sciences naturelles de Nimes, $n^{\circ} 5,1936,148 \mathrm{p}$.

BRAUN-BLANQUET (J.) et al.. - Les groupements vègètaux de la France méditerraneenne. - SIGMA, CNRS, 1952. - 289 p.

DEMANGEOT (J.). - Les milieux naturels du globe. - Paris: Masson, 1984. - 250 p.

DUCHAUFOUR (Ph.). - Stations, types d'humus et groupements écologiques. - Revue forestière française, vol. XII, $n^{\circ} 7,1960, p p .484-494$. 
DUVIGNEAUD (P.). - La synthèse écologique. - $2^{e}$ édition. - Paris : Doin, 1980. - $380 \mathrm{p}$.

ELLENBERG (H.). - Zeigerwerte des Gefäßpflanzen Mitteleuropas. - Zweite Auflage. - Scripta Geobotanica, vol. IX, 1979, pp. 1-122.

EMBERGER (L.). - Un projet de classification des climats du point de vue phytogéographique. - Bulletin de la Socjete d'Histoire naturelle de Toulouse, 77, 1930, pp. 97-124.

ESCARRE (J.), HOUSSARD (C.), DEBUSSCHE (M.). - Évolution de la végétation et du sol après abandon cultural en région méditerranéenne : étude de succession dans les garrigues du Montpellierais (France). Oecologia Plantarum, vol. 4, 18.3, 1983, pp. 221-239.

GODRON (M.). - Abrégé d'écologie de la végètation terrestre. - Paris: Masson, 1984. - $197 \mathrm{p}$.

GRECO 130043. - Rapport d'activité 1984-86. - Marseille: Université de Droit, Économie, Sc. d'AixMarseille III. - 148 p.

GRIME (J.P.). - Evidence for the existence of three primary strategies in plants and its relevance to ecological and evolutionary theory. - American Naturalist, $n^{\circ} 982,1977$, pp. 1169-1194.

MADJIDIEH (H.). - Caractéristiques biométriques et optiques du Chêne vert Quercus ilex (s.l.) dans différentes situations écologiques du Sud-Est méditerranéen français (Var). - Université d'Aix-Marseille III, 1987. $196 \mathrm{p},+$ annexes (Thèse de Doctorat).

MIGLIORETTI (F.). - Phytoécologie des peuplements à Quercus ilex L. et Quercus pubescens Willd. en Gardiole de Rians (Var). Approche méthodologique pour évaluer la phytomasse des taillis de Chêne vert. Faculté des Sciences d'Aix-Marseille III, 1983. - $77 \mathrm{p}$. + annexes (Thèse de $3^{\circ}$ cycle).

MIGLIORETTI (F.). - Écologie et dendrométrie des peuplements purs et mélangés de Chêne vert (Quercus ilex L.) et Chêne pubescent (Quercus pubescens Willd) en Provence. Bases méthodologiques, modèles de croissance et de production (application forêt de la Gardiole de Rians, Var). - Universitè d'Aix-Marseille Iil, 1987. - $292 \mathrm{p}$. (Thèse de Doctorat).

MIGLIORETTI (F.), BARBERO (M.). - Étude comparée de la densité des peuplements de Chêne vert en relation avec leur composition floristique: application à la forêt domaniale de la Gardiole de Rians (Var). Ecologia mediterranea, vol. $\mathrm{X}, \mathrm{n}^{\circ} 1-2,1984$, pp. 205-230.

MILLER (P.C.). - Some bioclimatic and pedologic influences on the vegetation in the mediterranean type region of South Africa. - Ecologia mediterranea, vol. VIII, $n^{\circ} 1-2,1982$, pp. 143-152.

ODUM (E.P.). - The strategy of ecosystem development. - Science, $\mathrm{n}^{\circ} 164,1969$, pp. 262-270.

PICKETT (S.T.A.). - Population pattern through 20 years of old field succession. - Vegetatio, $n^{\circ} 49,1982$, pp. $45-59$.

PONS (A.), VERNET (J.L.). - Une synthèse nouvelle de l'histoire du Chêne vert (Quercus ilex L.). - Bulletin de la Société botanique de France, vol. 118, 1971, pp. 841-850.

QUEZEL (P.), BARBERO (M.). - A propos des forêts de Quercus ilex L. dans les Cévennes. - Bulletin de la Société linnéenne de Provence, $n^{\circ} 38,1986$, pp. 101-117.

THINON (M.). - Incidence écologique des reboisements du Mont-Ventoux (Vaucluse). Aspects floristiques et pédologiques. - Université d'Aix-Marseille III, 1979. - 117 p. (Thèse).

VIGNES (A.). - Relation entre la surface prospectée et le nombre d'espèces végétales dans les peuplements naturels. - Annales de la Société des Sciences naturelles et archéologiques de Toulon, $n^{\circ} 32,1980$, pp. $102-119$.

YACINE (A.). - Une étude d'organisation génétique inter-et intrapopulation chez le Chêne vert (Quercus ilex). - Marseilie: Université des Sciences et Techniques de Languedoc, 1987, pp. 1-75 (Thèse de Spécialité). 